\title{
Comparison of Traditional and Integrated Bridge Design with SAGAT
}

\author{
F. Motz, E. Dalinger, H. Widdel \& S. Hockel \\ Research Institute for Communication, Information Processing and Ergonomics, \\ Wachtberg, Germany
}

S. MacKinnon

Memorial University of Newfoundland, St. John's, Canada

\begin{abstract}
Modular Integrated Navigation Systems (INS) according to the revised IMO performance standards on INS combine and integrate the validated information of different sensors and functions and allow the presentation on the various displays according to the tasks. The aim of the investigations discussed within this paper was to assess the advantages of an INS design compared to a traditional bridge layout with respect to the execution of collision avoidance and route monitoring tasks. The Situation Awareness Global Assessment Technique (SAGAT) approach was applied to assess Situation Awareness (SA) during these operations while bridge design and out of window visibility were manipulated. Additionally, workload was measured by NASA-TLX with its six subscales. The experiment was conducted in a full immersive simulation environment with 26 experienced mariners. The results indicate that SA is significantly higher with the INS bridge design in the reduced visibility condition compared to the traditional bridge design. Also, tendencies were found that workload and subdimensions are influenced by bridge design and visibility conditions.
\end{abstract}

\section{INTRODUCTION}

Modern ship bridges are highly-automated manmachine systems. Safety and efficiency of the ship operations are dependent upon the ability of a watchkeeper to perceive, interpret, and make decisions upon information acquired from the surrounding environment. In the last years a strong increase of modern information systems on ship bridges could be observed. Simple displays and control systems were supplemented or replaced by complex computer-based information systems. In order to support the mariner effectively onboard, a task- and situation-dependent representation of the information is a compelling need. Modular Integrated Navigation Systems (INS) according to the revised IMO performance standards on INS (IMO 2007) combine and integrate the validated information of different sensors and functions and allow the presentation on the various displays according to the tasks.

The aim of the investigations discussed within this paper was to assess the advantages of an INS design compared to a traditional bridge layout with respect to the execution of collision avoidance and route monitoring tasks. The Situation Awareness Global Assessment Technique (SAGAT) (Endsley 2000) approach was applied to assess Situation Awareness (SA) during these operations while bridge design and out of window visibility were manipulated. The method to assess the SA of watchkeeping officers on ship bridges was developed based on previous studies (Motz et al. 2008).

The experiment was carried out in the full mission bridge simulator of the Centre for Marine Simulation (CMS) of Memorial University of Newfoundland, St. John's, Canada. The experimental trials were conducted in a full bridge environment and carried out with four scenarios, to investigate the influence of bridge design and outside visibility on the SA of watchkeeping officers. The subjects were tasked to navigate a vessel in scenarios with varying traffic situations. In the trials a watch hand over was simulated so that after the first 10 minutes of monitoring and evaluating the traffic situation the subject assumed full control of the vessel. 


\section{SIMULATOR AND BRIDGE DESIGN}

The experiment was carried out in a 6 degree of freedom motion base, full mission ships bridge simulator (see Fig. 1). The simulator was manufactured by Kongsberg Maritime Ship System. All trials were conducted under a repeated motion profile. In the experiment two bridge configurations were compared: a traditional layout employing the existing navigational equipment and an INS design. The major difference between the two designs is the factor of integration of the collision avoidance and navigation information, including route monitoring and planning. The arrangement, location and design of the equipment of both configurations were identical.

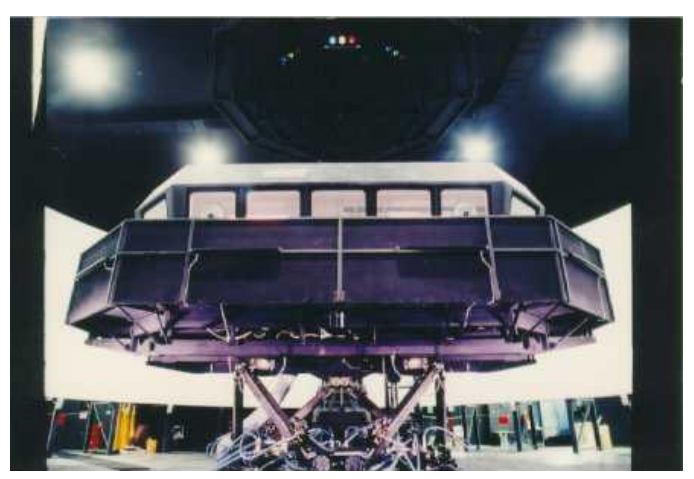

Figure 1. Full mission ships bridge simulator.

The following set up was used as traditional configuration:

- radar with facilities to display tracked radar targets

- electronic Charting Display and Information System (ECDIS) with route information

- minimum keyboard Display (MKD) to display AIS target information

- depth sounding information

- heading information

- speed information

- VHF communication (Navtex, VHF DSC)

- propulsion status displays

- steering and engine control

- steering status displays

- alarm information presented on the individual equipment

- chart table with paper charts.

For the INS configuration the information of the various navigational systems were integrated and combined in the displays on the bridge:

- collision avoidance display: radar with tracked radar targets and AIS targets, possibility to underlay ENC chart information

- route monitoring display: ECDIS with active route and AIS targets

- conning display: position, propulsion information, rate of turn, relative wind speed and direction, engine alarm status, rudder indicator, gyro repeater and speed
- minimum keyboard Display (MKD) to display AIS target information

- speed information

- VHF communication (Navtex, VHF DSC)

- propulsion status displays

- steering and engine control

- steering status displays

- alarm information presented on the individual equipment

- chart table with paper charts.

\section{SAGAT}

Situation Awareness is generally understood as "knowing what is going around you". Within the research community the definition of Endsley (1995) has been widely accepted in various domains. In a cognition-oriented approach, the model considers three levels and includes:

- perception of elements,

- comprehension of the meaning of the elements and the situation, and the

- projection of the status of the elements and the situation into the immediate future.

According to this model, decision making and performance is influenced by SA.

The Situation Awareness Global Assessment Technique (SAGAT) is probably one of the most well known SA measuring techniques (Endsley 2000). It provides an objective explicit measure of SA by directly comparing the operator's SA to an operational "scenario". With this technique, a simulation is frozen at a specific time, the system displays are blanked while the operator quickly has to answer questions concerning the scenario. Temporary freezes in the simulation must be of a short duration to minimise intrusiveness and memory decay. To get an accurate measure of the operator's SA the SAGAT probes must cover all three levels of SA and must be reflective of a wide range of the SA requirements. These are delineated through a goaldirected task analysis.

Table 1. Samples of SA questions.

\begin{tabular}{ll}
\hline \multirow{2}{*}{ Level } & Question \\
\hline \multirow{3}{*}{ Perception } & $\begin{array}{l}\text { What is the current position of your ves- } \\
\text { sel? }\end{array}$ \\
\cline { 2 - 2 } & $\begin{array}{l}\text { What is the course of the blue highlighted } \\
\text { vessel? }\end{array}$ \\
\hline \multirow{3}{*}{ Comprehension } & What is the distance to the next waypoint? \\
\cline { 2 - 2 } & $\begin{array}{l}\text { What is the direction of the course change } \\
\text { of the highlighted vessel? }\end{array}$ \\
\hline \multirow{2}{*}{ Projection } & $\begin{array}{l}\text { What is the CPA of the blue highlighted } \\
\text { vessel? }\end{array}$ \\
\cline { 2 - 2 } & $\begin{array}{l}\text { In how many minutes will you reach the } \\
\text { pilot station? }\end{array}$ \\
\hline
\end{tabular}


The method originally was developed for the aviation domain (e.g. Endsley 1990, Strater \& Endsley 2000) and has been applied in identical or modified forms in other domains. Presently, it was adapted to the marine domain (Motz et al. 2008). A sample of SAGAT probes for marine application is shown in Table 1.

SAGAT can be a useful tool to evaluate system design. SAGAT can provide a form of diagnostic information that indicates how a technology's design could improve or weaken an operator's SA when compared to a baseline technology. This information can then be used to refine design concepts. For the ergonomic evaluation of the task- and situation orientated presentation of navigational information on INS it was considered to use the concept of situation awareness measured with SAGAT.

Questions related to perception of elements (level 1) refer to the status of own ship as well as dynamics of relevant objects in the environment. A mariner has to possess correct information of own ship (position, route, course etc.) as well as correct information about targets (speed, distance etc.).

Questions related to comprehension of meaning (level 2) go beyond simply being aware of the elements that are presented. An understanding of the significance of those elements in light of mariner's goals is included. For example, a mariner must quickly determine those targets which pose a threat and eventually demand operator action to mediate a threat or obstacle.

Questions related to projection of the near future (level 3) refer to future actions of the elements in the environment. This is achieved through knowledge of the status and dynamic of the elements and a comprehension of the situation.

\section{EXPERIMENT}

\subsection{Subjects}

26 experienced mariners (masters, navigational officers, pilots) participated in the trials. The requirements for subject recruitment were:

- at least half a year experience as officer of the watch (OOW)

- the mariners must have navigated a vessel in the last four years

- or working actively as navigational simulator instructors.

\subsection{Hypotheses}

It was hypothesized that navigating with INS bridge design leads to higher SA scores than navigating with traditional bridge design. This effect might be more distinctive under difficult conditions like reduced outside view when fog prevails than under good view. In the case of bad navigation conditions also mental workload may be increased.

\subsection{Experimental Design}

A 2 x 2 factorial design with two within-subject factors was used. The first within-subject factor bridge design varied on the two levels:

- traditional configuration

- INS configuration.

- The second within-subject factor visibility varied on the two levels:

- good visibility

- reduced visibility (fog).

Dependent variables were SA and NASA-TLX scores (Hart \& Staveland 1988).

A $2 \times 2$ repeated measures ANOVA (Analysis of Variance) model with the within subject factors bridge design and visibility were employed to analyse these data. As dependent variables the relative frequency of correct answers representing the SA score were analyzed. For workload the NASA-TLX scores were used. All statistical interpretations were considered at the $5 \%$ level of significance.

\subsection{Experimental Control}

The experiment was carried out in the ships bridge simulator with the two bridge designs described in Section 2.

To control the presentation of SA questions on the bridge, to log the answers of the subjects and to monitor the vessels involved in a scenario an experimental controlling program was developed by Research Institute for Communication, Information Processing and Ergonomics. The program consists of the question presentation module, the nautical chart module presenting the graphical information for the SA questions and the experimental control module (see Fig. 2). The three different modules were located on different computers in the network. Whereas the nautical chart module and the question presentation module were installed on the navigational bridge of the simulator, the experimental control module runs on a PC in the simulator control room.

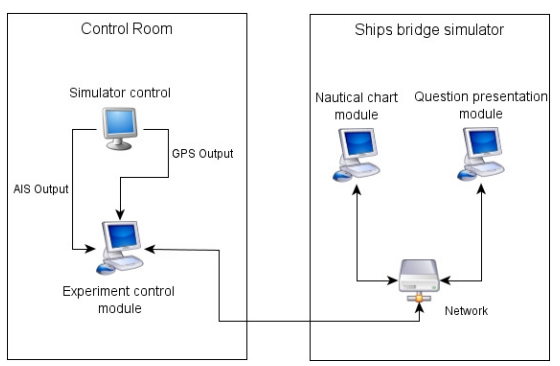

Figure 2. Controlling components of experiment. 
The experimental control module fulfilled the three main tasks processing of simulator output, target control and control of SA questioning.

The experimental control module read and processed the data of own ship's GPS output signal and the AIS signals of the other vessels provided to the navigational equipment (e.g., the radar, ECDIS or the MKD) on the bridge. This allowed the SA questions to be posed using real-time data. Therefore, the software offered the possibility to show during the simulation the movement of own ships and the other vessels on a chart background and to offer suggestions to change the course or speed of the vessels in case of course or speed alterations of the own ship in order to present the SA questions in similar traffic situations for all participants.

\subsection{Presentation of SA questions on the bridge}

When the scenarios were "frozen" to present the subjects the SA questions, the simulation was suspended, all task relevant information was removed from the navigational equipment on the bridge and the outside view was blanked. The SA questions were then administered on two displays on the bridge. Whereas on one of the displays the questions were presented, on the other display additional graphical information for certain questions were displayed (see Fig. 2).

This offered the four techniques to ask the SA questions, depending on the topic, complexity and the easiest way to present and to answer the questions:

- numeric open-end questions: Questions appeared on the question display as numeric open-end questions, e.g., what is your heading after the next waypoint. No graphical information was presented on the graphical information display. To answer the questions the subjects had to type in the right answer and then to click on the continue button to proceed with the next question

- open-end questions with graphical answer: The question after the position of the vessel appeared on the question display as open-end question (instruction) and had to be answered on the graphical information display by clicking on the chart background.

- numeric open-end questions with additional graphical information: Questions appeared on the question display as open-end questions with additional information presented on the chart of the graphical information display, e.g., the target involved in the question. To answer the questions the subjects had to type in the right answer and then click on the continue button to proceed with the next question
- multiple choice questions with additional graphical information: Questions appeared on the question display as multiple choice questions and on the graphical information display question related information was presented on the chart background. To answer the questions the subjects had to select the right answer and then to click on the continue button to proceed with the next question.

A preliminary question-pool of about 70 questions had been developed referring to the navigation of the vessel focusing on route monitoring and collision avoidance. The questions were evaluated in pretests which had the aim of selecting the most relevant questions, of testing the content and understandability, and to reduce the number of questions resulting in a set of 16 questions on three levels (see Table 1 for examples).

\subsection{Traffic scenarios}

The SAGAT approach required the development of realistic scenarios based on specific criteria, e.g., course change of own ship, navigational hazards, traffic density, and "interest/danger" of targets. The criteria for traffic density and "interest" of targets are:

- total number of targets - 20

- number of targets within a 3 NM range - 10

- number of targets within a $3 \mathrm{NM}$ range: close quarter targets (CPA: $0.5-1.5 \mathrm{NM}$ ); with a collision course, overtaking own ship or overtaken by own ship - 5

- number of targets, which cause a reaction because of collision course - 1 .

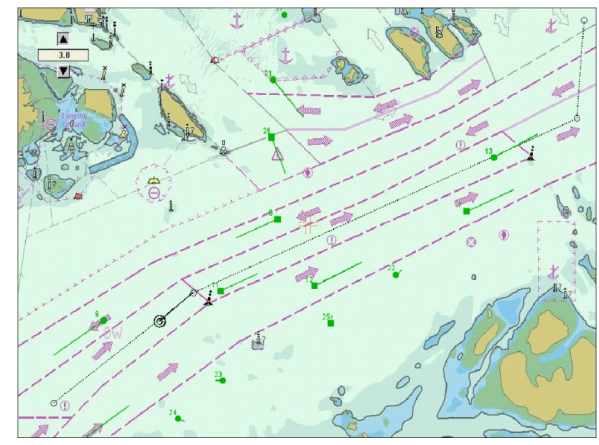

Figure 3: Singapore scenario at the first freezing.

On the basis of these criteria four traffic scenarios with duration of 21.5 - 25 minutes were developed, as well as one familiarization/habituation scenario. To ensure that the previous knowledge of traffic and of the sea area doesn't influence the results (i.e. a learning effect was controlled for), the scenarios represent different traffic situations for the Juan de Fuca Strait / Strait of Georgia (familiarization scenario), English Channel and Singapore. Figure 3 shows one Singapore scenario at the time of the first freezing. In the pre-trials the traffic scenarios were evaluated in respect to realism, relevance and complexity. 


\subsection{Experimental procedure}

The experimental procedure had five steps.

In the first step the experiment was introduced to the subjects in a briefing outside the simulator. Subjects had to complete a personal data sheet, which was used to gather data like current occupation, years of experience as mariner, and age, and the intention of the experiment and general description of experimental set up were described to the subjects.

In the second step subjects were familiarized in the simulator with the experimental procedure and the bridge equipment. The INS design and the traditional layout were explained in detail and the procedure with the presentation of scenarios and the following interruptions for SA questioning were explained.

In the third step familiarization trials were conducted, one familiarization trial for each bridge layout. The purpose was to familiarize the subject with the navigation of the vessel with the different bridge layouts, with the experimental procedure of the freezings and with the different types of SA questions. The familiarization trials were carried out without motion and with good visibility for all trials.

In the next step the four scenarios were presented to the subject in a randomized order. The task of the subject was to navigate a vessel in traffic situations of varying density with either good or reduced visibility. In the trials a watch hand over was simulated so that the first 10 minutes of each scenario the subject monitored and evaluated the traffic situation. An instructor was fulfilling the role of the officer of the watch for the first 10 minutes. After the hand over, the subject was in full control of the vessel. An instructor remained on the bridge and acted as both the helmsman and the master. Thus, as the helmsman, the instructor performed any changes in speed and course and as master, to deny inappropriate decisions of the subject that might disrupt the whole experiment.

During each scenario there happened four freezings in which the outside view and the displays were blanked and the SA questions were asked. The first freezing was conducted at the watch hand over and the last at the end of the scenario. Same questions were asked for all treatments, 16 questions per scenario divided into 4 groups of 4 questions.

At the end of each scenario the NASA-TLX rating scale was completed. Following the collection of all four scenarios the NASA-TLX rating paired comparisons questionnaire were completed.

The duration of a simulation trial (4 scenarios and habituation) per subject was between 190 to 220 minutes, depending on the time each subject needed to become familiarize with the bridge equipment. The trials were carried out with motion.

After the trials a SAGAT debriefing questionnaire and a INS questionnaire to evaluate certain aspects of an INS layout were administered to the subjects in a separate room.

\section{RESULTS}

\subsection{Main results}

The central questions of the experiment were focused on the impact of the independent variables, bridge design and visibility, on situation awareness. The means of frequencies of correctly answered SA questions are shown in Figure 4. The results of the ANOVA for the within subject factors bridge design and visibility show a significant main effect for the factor bridge design $\left(\mathrm{F}_{1,25}=4.88, \mathrm{p}<0.05\right)$ and $\mathrm{a}$ significant interaction effect between bridge design and visibility $\left(\mathrm{F}_{1,25}=6.94, \mathrm{p}<0.05\right)$. No significant effect for the factor visibility $\left(\mathrm{F}_{1,25}=0.94, \mathrm{p}>0.3\right)$ could be found.

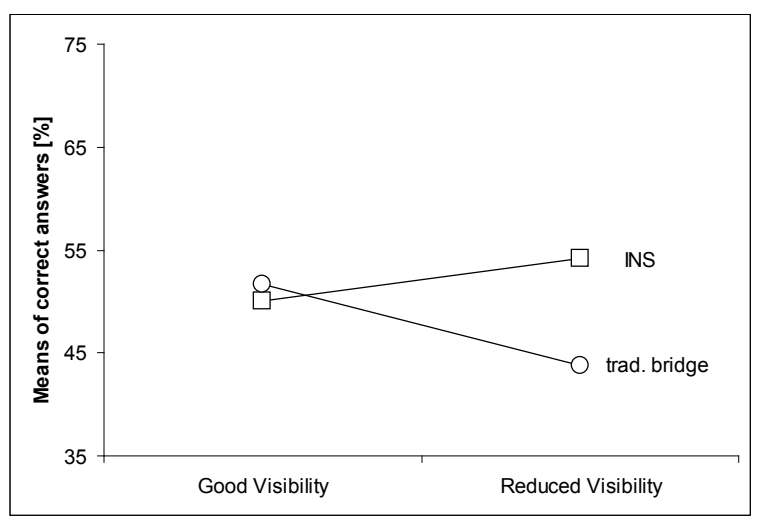

Figure 4. Dependency of SA from bridge design and visibility.

The analysis of variance executed for the overall workload, defined by the total score of NASA-TLX, does not result in significant differences for the two main effects visibility $\left(\mathrm{F}_{1,25}=3.57, \mathrm{p}=0.07\right)$ and bridge design $\left(\mathrm{F}_{1,25}=1.01, \mathrm{p}=0.32\right)$ or for the interaction $\left(\mathrm{F}_{1,25}=0.13, \mathrm{p}=0.72\right)$. But a strong tendency can be seen that the INS produces less workload than a traditionally designed bridge, and reduced visibility is responsible for higher workload. Although, tendencies were found for the subscales performance and effort favoring the INS bridge design especially under the condition of reduced visibility.

Results from the INS questionnaire support the empirical data collected in this study. In general, the majority of the subjects (93\%) preferred the INS bridge design compared to traditional design. The participants who preferred the traditional bridge gave as reasons that they are more used to the traditional design and that the INS design provides too much information. As added value of an INS almost 
all participants chose the answer "the combination of information" (see Fig. 5). Half of the respondents selected "the integrity of data" (meaning the possibility to compare automatically data from independent sources). Less often selected is "the higher quality of information".

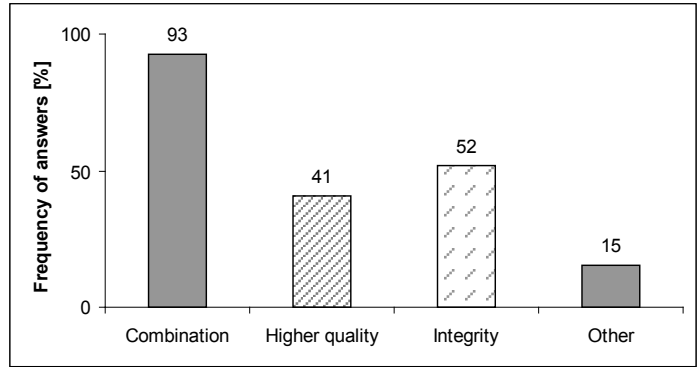

Figure 5. Means of frequency for the added value of an INS bridge.

\subsection{Post-hoc analysis}

The definition of SA specifies a hierarchical structure with three levels (see Section 3). Questions for the first level are preconditions for answering questions on level 2 and 3. Following this hierarchical organization leads to the assumption that questions of level 1 are answered correctly more often than questions of the higher levels.

In the post-hoc analysis the factor level of $S A$ was included. A $2 \times 2 \times 3$ ANOVA with the factors bridge design, visibility and level of $S A$ as within subject factors was performed to test the assumption which was justified by a significant main effect of the factor level of $S A\left(\mathrm{~F}_{2,50}=43.47, \mathrm{p}<0.001\right)$.

A pairwise comparisons with Bonferroni correction of the three SA level scores show a significant higher score for level 1 compared to level 2 and level 3, but no difference between the latter two. In Figure 6 the means of frequencies of correct answers for the 3 levels of SA are shown for condition reduced visibility.

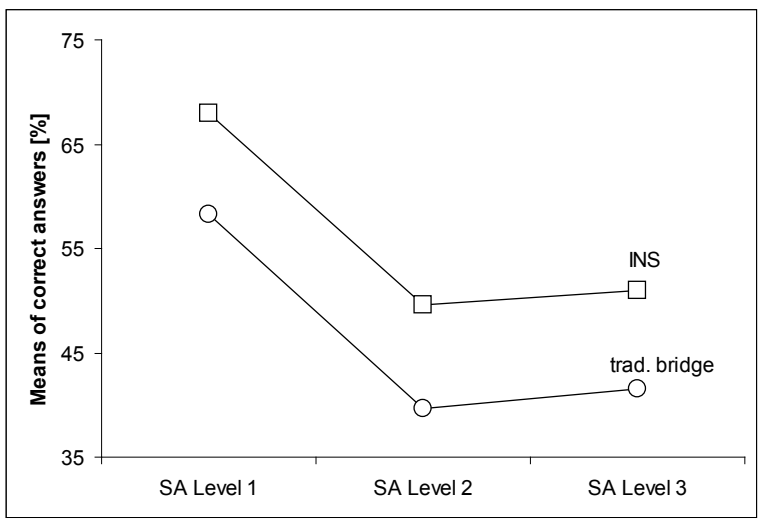

Figure 6. Dependency of SA from bridge design on the three SA levels.

SA level 1 (perception) had a greater score than SA levels 2 and 3 (comprehension and projection), suggesting that the application of SAGAT in this maritime-related research was a valid approach to assess global SA.

\section{CONCLUSIONS}

The results indicate that bridge design has a significant impact on the degree of situation awareness, as hypothesized. SA is significantly higher with the INS bridge design in the reduced visibility condition compared to the traditional bridge design. In good visibility the SA is similar with both bridge designs. Mariners have to rely more on information systems when navigating in reduced visibility conditions. It can be hypothesized that not only reduced visibility but detrimental navigational conditions, in general, may reduce SA when navigating with traditionally designed bridges but not with INS. These considerations also apply to workload in the sense that stress inducing work conditions can influence total workload and subdimensions of NASA-TLX like performance and effort when using traditional bridge design.

Further experiments are required comprising more difficult navigation surroundings, e.g., higher traffic density, more challenging navigation tasks, high stress inducing work environment, to confirm and sharpen these experimental findings.

\section{REFERENCES}

Endsley, M.R. 1990. A methodology for the objective measurement of situation awareness. In Situational Awareness in Aerospace Operations (AGARD-CP-478): 1-9. Neuilly sur Seine, France: NATO- AGARD.

Endsley, M.R. 1995. Toward a theory of situation awareness in dynamic systems. Human Factors 37: 32-64.

Endsley, M.R. 2000. Direct Measurement of Situation Awareness. In M.R. Endsley \& D.J. Garland (eds.), Situation Awareness Analysis and Measurement: 147-173. Mahwah, NJ: Lawrence Erlbaum Associates.

Hart, S.G. \& Staveland, L.E. 1988. Development of NASATLX (Task Load Index): Results of empirical and theoretical research. In P. Hancock \& N. Meshkati (eds.), Human Mental Workload: 139-183. Amsterdam: North Holland.

IMO 2007. Revised performance standards for integrated navigation systems (INS). MSC.252(83). London: International Maritime Organization.

Motz, F., MacKinnon, S., Dalinger, E., Widdel, H. 2008. Application of SAGAT for Navigational Tasks on Ship Bridges. International Maritime Conference / ABCD Symposium on Human Performance at Sea. Sydney, Australia.

Strater, L. \& Endsley, M.R. 2000. SAGAT- A situation awareness measurement tool for commercial airline pilots. In D.B. Kaber \& M.R. Endsley (eds.), Proceedings of the first human performance, situation awareness, and automation: User-centered design for the new millennium conference: 360. Savannah, GA. 